\title{
Etapas del Proceso Administrativo
}

\author{
Stages the administrative process \\ Jorge Martín Hernández Mendoza ${ }^{a}$, Sandra Luz Hernández Mendoza ${ }^{b}$
}

\begin{abstract}
:
Administration as a science involves in its theoretical and practical application a systematized scheme that breaks with improvisations that promote thought in research.

The administrative process is a model developed by Henry Fayol, which has been identified as the fundamental basis of administrative practice giving it a broader capacity for abstraction and the possibility of generating theoretical concepts increasingly specific to the needs of the companies, being also a model with which the administrator function is standardized.
\end{abstract}

The administrative process is defined as the achievement of phases or stages through which the administrative practice is carried out. Therefore, at present, the quadripartite division is the most universally accepted: planning, organization, direction and control.

Considering the importance of these stages and / or phases, it can be said that the administrative process becomes the principle of any administrative activity, whose main objective is the systematization of knowledge generating a structure of efficiency, as well as fostering the development of a philosophy and managerial culture (Varela \& León 2010).

The stages and / or phases of the administrative process propose methods developed based on the experience of large companies where:

Planning points out the need to set objectives, it is considered the most important stage that implies a rational decision making.

The organization seeks to meet the objectives established in the planning.

The management focuses its attention on how to delegate authority and coordinate activities.

The control establishes the criteria that must be applied in the measurement and evaluation of results.

\section{Keywords:}

Organization, efficiency, administration

\section{Resumen:}

La administración como ciencia involucra en su aplicación teórica y práctica un esquema sistematizado que rompe con improvisaciones que promueven el pensamiento en la investigación.

El proceso administrativo es un modelo desarrollado por Henry Fayol, al cual se le ha identificado como la base primordial de la práctica administrativa otorgándole a ésta una capacidad de abstracción más amplia y la posibilidad de generar conceptos teóricos cada vez más particularizados a las necesidades de las empresas, siendo también un modelo con el cual se estandariza la función del administrador.

El proceso administrativo se define como una consecución de fases o etapas a través de las cuales se lleva a cabo la práctica administrativa. Por eso, en la actualidad, la división cuatripartita, es la más aceptada universalmente: planeación, organización, dirección y control.

Considerando la importancia de estas etapas y/o fases se puede decir que, el proceso administrativo se convierte en el principio de cualquier actividad administrativa, cuyo objetivo principal es la sistematización del conocimiento generando una estructura de eficacia, además de fomentar el desarrollo de una filosofía y cultura gerencial (Varela \& León 2010).

Las etapas y/o fases del proceso administrativo proponen métodos desarrollados con base a la experiencia de las grandes empresas donde:

\footnotetext{
a Universidad Autónoma del Estado de Hidalgo, Escuela Superior de Atotonilco, ORCID0000-0002-9262-4712, Email: jorge_hernandez6100@uaeh.edu.mx

${ }^{\mathrm{b}}$ Universidad Autónoma del Estado de Hidalgo, Instituto de Ciencias Básicas e Ingeniería, ORCID: 0000-0002-2022-3135, Email: sandrahm@uaeh.edu.mx
} 
La planeación señala la necesidad de fijar objetivos, se considera la etapa más importante que implica una racional toma de decisiones. La organización busca cumplir los objetivos establecidos en la planeación.

La dirección centra su atención en la manera de delegar autoridad y coordinar actividades.

El control establece los criterios que se deben aplicar en la medición y evaluación de resultados.

\section{Palabras Clave:}

Organización, eficiencia, administración

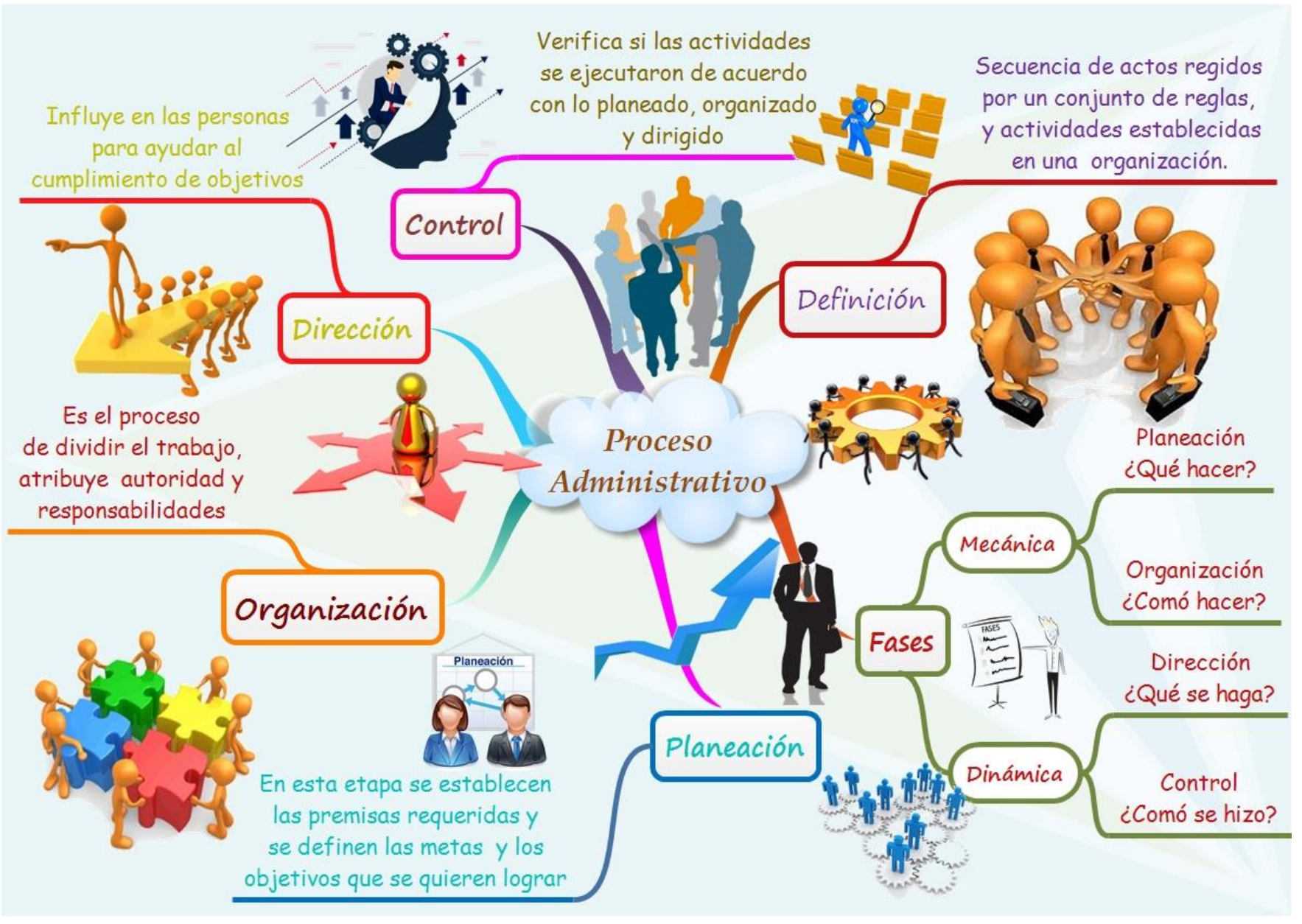

\section{Referencias}

[1] Reyes, A. (2007). Administración moderna. Editorial Limusa, grupo Noriega editores, recuperado y consultado el 18 de agosto de 2018 de: https://educativoinsurgentes.files.wordpress.com/2018/01/administracion-moderna-reyes-ponce.pdf

[2] Varela, M., y León, A. (2010). La evolución del proceso administrativo frente al marco metodológico de la creación de valor como principio rector de la gestión empresarial. Ciencia económica, 28, Núm. 2, pp. 465-480, recuperado y consultado el 18 de agosto de 2018 de: http://brd.unid.edu.mx/la-evoluciondel-proceso-administrativo.html 